\title{
A particle model with extra dimensions from coadjoint Poincaré symmetry
}

\author{
Andrea Barducci, ${ }^{a}$ Roberto Casalbuoni ${ }^{a}$ and Joaquim Gomis $^{b}$ \\ ${ }^{a}$ Department of Physics and Astronomy, University of Florence and INFN, \\ Via G. Sansone 1, Sesto Fiorentino 50019, Firenze, Italy \\ ${ }^{b}$ Departament de Física Quàntica i Astrofísica and Institut de Ciències del Cosmos (ICCUB), \\ Universitat de Barcelona, Martí i Franquès, Barcelona ES-08028, Spain \\ E-mail: barducci@fi.infn.it, casalbuoni@fi.infn.it, gomis@ecm.ub.es
}

ABstract: Starting from the coadjoint Poincaré algebra we construct a point particle relativistic model with an interpretation in terms of extra-dimensional variables. The starting coadjoint Poincaré algebra is able to induce a mechanism of dimensional reduction between the usual coordinates of the Minkowski space and the extra-dimensional variables which turn out to form an antisymmetric tensor under the Lorentz group. Analysing the dynamics of this model, we find that, in a particular limit, it is possible to integrate out the extra variables and determine their effect on the dynamics of the material point in the usual space time. The model describes a particle in $D$ dimensions subject to a harmonic motion when one of the parameters of the model is negative. The result can be interpreted as a modification to the flat Minkowski metric with non trivial Riemann, Ricci tensors and scalar curvature.

Keywords: Space-Time Symmetries, Global Symmetries

ARXIV EPRINT: 2006.11725 


\section{Contents}

1 Introduction 1

2 Maurer-Cartan one-forms 3

3 A model with extra-dimensions 4

4 Dimensional reduction $\quad 6$

4.1 Hamiltonian analysis and canonical action 8

$\begin{array}{ll}4.2 \text { The effective action } & 10\end{array}$

5 Conclusions and outlook 13

\section{Introduction}

The space-time symmetry algebra of non-relativistic string theory $[1,2]$ is the stringy Galilei algebra [3-6]. This algebra contains a vector and an antisymmetric two tensor "central" generators, and it can be obtained as a contraction of the coadjoint Poincaré algebra in $D$ dimensions [7] with generators $P_{\mu}$ and $M_{\mu \nu}$ and two abelian charges $Z_{\mu}$ and $Z_{\mu \nu}$. The numbers of generators of the algebra is $D(D+1)$.

A dynamical realization of the coadjoint Poincaré algebra in three dimensions has appeared in [8] where non-relativistic gravities in three dimensions [9-12] have been obtained as a non-relativistic limit from the coadjoint Poincaré gravity.

Here we will consider another realization of the coadjoint Poincaré algebra in any dimension constructing a particle action invariant under this symmetry. We will obtain the action using the non-linear realization approach to space-time symmetries.

We will consider the Maurer-Cartan (MC) one-forms, constructed by the quotient of the coadjoint Poincaré algebra with respect to the Lorentz group generators. The coset space is described by three types of coordinates, the Minkowski usual space-time coordinates, $x^{\mu}$ and the coordinates $\xi^{\mu \nu}$ and $\eta^{\mu}$ associated to the generators $Z_{\mu \nu}$ and $Z_{\mu}$ respectively. There are three MC forms associated to the generators in the coset and, in principle, this allows various kind of dynamical models, describing particles and, more generally, $p$-branes.

In this paper we will first concentrate on a particular particle model, using first the MC forms associated to the generators $P_{\mu}$ and $Z_{\mu \nu}$. We find that a combination of these forms describes a free particle model in a space-time of $D+D(D-1) / 2$ dimensions, parametrised by the coordinates $x^{\mu}$ and $\xi^{\mu \nu}$. It is interesting to notice that, in this case, the extra-dimensions are not parametrised by Lorentz scalars but by an antisymmetric tensor. 
The signature of the Minkowski space time metric is mostly plus, however the total space time is a space with more that one time. In $D=4$, the signature is $(1,3)$. Instead, the extended space time has dimensions 10 and the signature $(4,6)$, since the "electric" coordinates $\xi^{0 i}$ have negative signature. Of course, the model obtained in this way has a symmetry much larger that the original coadjoint Poincaré algebra. In fact, the symmetry group is the Poincaré group in $D(D+1) / 2$ dimensions whose generators are $D(D+1)(D(D+1)-2) / 8$, which is much larger than the generators of the coadjoint Poincaré algebra.

The coadjoint Poincaré symmetry offers a simple way to do a dimensional reduction, through the MC one-form associated to the generator $Z_{\mu}$. This form contains the coordinates $\eta^{\mu}$ and a coupling between the $x^{\mu}$ 's and the $\xi^{\mu \nu}$ 's. This coupling breaks the Poincaré symmetry in $D(D+1) / 2$ space-time dimensions to the coadjoint Poincare symmetry in $D$ dimensions that contains the Poincaré symmetry in the same number of space time dimensions.

The model depends crucially on two parameters, one, $z$ dimensionless, weighting the relevance of the extra dimensional variables $\xi^{\mu \nu}$ relatively to our space-time variables $x^{\mu}$. The other parameter $R$ with dimension of a length measures the relevance of the third $\mathrm{MC}$ form with respect to the first two. In other words, it measures the importance of the coupling between $x^{\mu}$ and $\xi^{\mu \nu}$. As we will show, the dynamics of the variables $\eta^{\mu}$ defines a constant time-like vector, $F^{\mu}$, which is the canonical momenta associated to $\eta^{\mu}$ written in terms of lagrangian variables. The dynamics allows to integrate the variables $\xi^{\mu \nu}$ in terms of $x^{\mu}$ in a particular limit where we send $R \rightarrow \infty$ and $z \rightarrow 0$ as $1 / R^{3}$. Therefore we can construct an effective action for $x^{\mu}$. This effective action breaks the coadjoint Poincaré symmetry of $D$ space time dimensions. The effective action describes a particle in $D$ dimensions subject to a harmonic motion, for $z<0$, on a $(D-1)$ subspace orthogonal to the constant time-like vector $F^{\mu}$. This gives rise to a modification of the mass of the particle which, in the first quantised version produces a tower of particle with mass spaced by the frequency of the oscillator, $\omega \sim 1 / \sqrt{R}$. We show that this situation can be also described as a modification to the flat Minkowski metric in $\mathrm{D}$ dimensions by a quadratic term in the coordinates transverse to the vector $F^{\mu}$. The modification of the flat metric is non trivial, giving rise to non zero Riemann, Ricci tensor and scalar curvature. There are other two limits, one sending $z \rightarrow 0$ with $R$ fixed. In this case we obtain a free particle in $D$ dimensions (see later). The other is $R \rightarrow \infty$ with fixed $z$ which describes a free particle in $D(D+1) / 2$ dimensions.

The paper is organised in the following way: in section 2 we introduce the coadjoint Poincaré algebra and we evaluate the three MC forms. In section 3 we discuss a model which can be interpreted as describing a free particle in $D(D+1) / 2$ dimensions and we analyse its symmetries. In section 4 we consider the dimensional reduction of the previous model through the addition of a second term to the action obtained from the third MC form. The resulting model is invariant under two distinct diffeomorphisms (diff). In section 4.1 we discuss the constraints induced by the diff-invariance. In section 4.2 we consider the limit where $z \rightarrow 0$ and $R \rightarrow \infty$ in a correlated way and proceed to integrate out the variables $\xi^{\mu \nu}$, obtaining an effective action. In section 5 we summarise the main results obtained in 
this paper and we discuss also some perspective as, for instance, the extension to strings and $p$-branes.

\section{Maurer-Cartan one-forms}

The coadjoint Poincaré algebra is an extension of the Poincaré algebra with a vector and an antisymmetric rank-two tensor, $Z_{\mu}$ and $Z_{\mu \nu}$ respectively, satisfying the commutation relations [7]

$$
\begin{aligned}
{\left[M_{\mu \nu}, Z_{\rho}\right] } & =i\left(\eta_{\mu \rho} Z_{\nu}-\eta_{\nu \rho} Z_{\mu}\right) \\
{\left[M_{\mu \nu}, Z_{\rho \sigma}\right] } & =i\left(\eta_{\mu \rho} Z_{\nu \sigma}+\eta_{\nu \sigma} Z_{\mu \rho}-\eta_{\mu \sigma} Z_{\nu \rho}-\eta_{\nu \rho} Z_{\mu \sigma}\right) \\
{\left[Z_{\mu \nu}, P_{\rho}\right] } & =i\left(\eta_{\mu \rho} Z_{\nu}-\eta_{\nu \rho} Z_{\mu}\right), \\
{\left[Z_{\mu}, P_{\nu}\right] } & =0, \quad\left[Z_{\mu}, Z_{\nu}\right]=0, \quad\left[Z_{\mu \nu}, Z_{\rho}\right]=0, \quad \mu, \nu=0,1 \cdots D-1
\end{aligned}
$$

Let us consider the quotient space of the group generated by this algebra with respect to the Lorentz group. A local parameterisation of the coset is given by

$$
g=e^{i x^{\mu} P_{\mu}} e^{\frac{i}{2} \xi^{\mu \nu} Z_{\mu \nu}} e^{i \eta^{\mu} Z_{\mu}}
$$

The Maurer Cartan (MC) one-form is given by

$$
\Omega=-i g^{-1} d g=d x^{\mu} P_{\mu}+\left(d \eta^{\mu}-\xi^{\mu \nu} d x_{\nu}\right) Z_{\mu}+\frac{1}{2} d \xi^{\mu \nu} Z_{\mu \nu}
$$

or:

$$
\Omega=\Omega_{1}^{\mu} P_{\mu}+\frac{1}{2} \Omega_{2}^{\mu \nu} Z_{\mu \nu}+\Omega_{3}^{\mu} Z_{\mu}
$$

where

$$
\Omega_{1}^{\mu}=d x^{\mu}, \quad \Omega_{2}^{\mu \nu}=d \xi^{\mu \nu}, \quad \Omega_{3}^{\mu}=d \eta^{\mu}-\xi^{\mu \nu} d x_{\nu} .
$$

It is easy to verify that the $\mathrm{MC}$ forms are invariant under the following transformations:

$$
\begin{aligned}
P_{\mu}: & \delta x^{\mu} & =a^{\mu} \\
Z_{\mu \nu}: & \delta \xi^{\mu \nu} & =\epsilon^{\mu \nu}, \quad \delta \eta^{\mu}=\epsilon^{\mu \nu} x_{\nu} \\
Z_{\mu}: & \delta \eta^{\mu} & =\epsilon^{\mu} .
\end{aligned}
$$

These transformations are generated by the right invariant vector fields

$$
P_{\mu}=-i \frac{\partial}{\partial x^{\mu}}, \quad Z_{\mu}=-i \frac{\partial}{\partial \eta_{\mu}}, \quad Z_{\mu \nu}=-i \frac{\partial}{\partial \xi^{\mu \nu}}+i\left(x_{\mu} \frac{\partial}{\partial \eta^{\nu}}-x_{\nu} \frac{\partial}{\partial \eta^{\mu}}\right) .
$$

Furthermore the Lorentz group generators are

$$
M_{\mu \nu}=-i\left(x_{\mu} \frac{\partial}{\partial x^{\nu}}-x_{\nu} \frac{\partial}{\partial x^{\mu}}\right)-i\left(\eta_{\mu} \frac{\partial}{\partial \eta^{\nu}}-\eta_{\nu} \frac{\partial}{\partial \eta^{\mu}}\right)-i\left(\xi_{\mu \rho} \frac{\partial}{\partial \xi_{\rho}^{. \nu}}-\xi_{\nu \rho} \frac{\partial}{\partial \xi_{\rho}^{\cdot \mu}}\right) .
$$




\section{A model with extra-dimensions}

Starting from the MC forms given in (2.5) and using the non-linear realization approach to space-time symmetries, one can construct many actions invariant under the coadjoint Poincaré algebra. Here we will consider an action involving only the coordinates $x^{\mu}, \xi^{\mu \nu}$ through the pullback of two MC forms $\Omega_{1}^{\nu}$ and $\Omega_{2}^{\mu \nu}$ :

$$
S_{1}=-M \int d \tau \sqrt{-\dot{x}_{\mu}^{2}-\frac{z}{2} \dot{\xi}_{\mu \nu}^{2}}
$$

The sign of the parameter $z$ is simply related to the sign with which the "electric", $\xi^{0 i}$, and the "magnetic" components, $\xi^{i j}$, contribute to the line element in (3.1).

We assume also the following dimensions in mass:

$$
\left[x^{\mu}\right]=\left[\xi^{\mu \nu}\right]=-1 .
$$

Let us nos consider the global symmetries of this lagrangian. Since the $\eta_{\mu}$ variables do not appear, we can eliminate all this dependence on the generators of eqs. (2.7) and (2.8). It follows that, for the moment being, the symmetries generated by $Z_{\mu}$ can be simply ignored. Also, the variables $x_{\mu}$ and $\xi_{\mu \nu}$ are decoupled. Therefore, the model is invariant under two independent Lorentz groups, one acting on the position variables and the other on $\xi_{\mu \nu}$. It is convenient to define the following quantities:

$$
M_{\mu \nu}^{1}=-i\left(x_{\mu} \frac{\partial}{\partial x^{\nu}}-x_{\nu} \frac{\partial}{\partial x^{\mu}}\right)
$$

and

$$
M_{\mu \rho, \sigma \nu}^{2}=-\frac{i}{2}\left(\xi_{\mu \rho} \frac{\partial}{\partial \xi^{\sigma \nu}}-\xi_{\sigma \nu} \frac{\partial}{\partial \xi^{\mu \rho}}\right) .
$$

Notice that the part of the original Lorentz group generators, excluding the $\eta_{\mu}$ part, is given by

$$
M_{\mu \nu}^{1}+2 \eta^{\rho \sigma} M_{\mu \rho, \sigma \nu}^{2} .
$$

We see that $L_{1}$ is also invariant under the translations in $x_{\mu}$ and $\xi_{\mu \nu}$ generated by $P_{\mu}$ and $Z_{\mu \nu}$ respectively. That is to say, our lagrangian is invariant under the two Poincaré groups acting on $x^{\mu}$ and $\xi^{\mu \nu}$. In 4 dimensions we have $10+21=31$ symmetries.

However the full symmetry group of $L_{1}$ is much larger. In fact introducing the following variables

$$
y^{A}=\left(x_{\mu}, \sqrt{\frac{|z|}{2}} \xi^{\mu \nu}\right)
$$

we can write $L_{1}$ as a lagrangian for a free particle in $D(D+1) / 2$ dimensions:

$$
L_{1}=-M \sqrt{-\eta_{\mu \nu} \dot{x}^{\mu} \dot{x}^{\nu}-\frac{z}{2} \eta_{\mu \rho} \eta_{\nu \sigma} \dot{\xi}^{\mu \nu} \dot{\xi} \rho \sigma}=-M \sqrt{-\eta_{A B} \dot{y}^{A} \dot{y}^{B}}
$$

with

$$
\eta_{A B}=\left(\eta_{\mu \nu}, \epsilon(z) \eta_{\mu \rho} \eta_{\nu \sigma}\right)
$$




\begin{tabular}{|c|c|c|c|}
\hline generators & dimensions & $\mathrm{D}=3$ & $\mathrm{D}=4$ \\
\hline$P_{A}$ & $D(D+1) / 2$ & 6 & 10 \\
\hline$P_{\mu}$ & $D$ & 3 & 4 \\
\hline$Z_{\mu \nu}$ & $D(D-1) / 2$ & 3 & 6 \\
\hline
\end{tabular}

Table 1. Translation generators.

\begin{tabular}{|c|c|c|c|}
\hline generators & dimensions & $D=3$ & $D=4$ \\
\hline$S_{A B}$ & $D(D+1)(D(D+1)-2) / 8$ & 15 & 45 \\
\hline$S_{\mu \nu}$ & $D(D-1) / 2$ & 3 & 6 \\
\hline$S_{\mu \nu ; \rho \sigma}$ & $D(D-1)(D(D-1)-2) / 8$ & 3 & 15 \\
\hline$S_{\mu \nu ; \rho}$ & $D(D(D-1) / 2$ & 9 & 24 \\
\hline
\end{tabular}

Table 2. Lorentz generators.

In the particular case of $D=4$ this is the lagrangian of a free particle moving in 10 dimensions.

The symmetry algebra of $L_{1}$ is the Poincaré algebra in $D+D(D-1) / 2$ dimensions. For example, for $D=4$, the Poincaré group in 10 dimensions has 55 generators. In general, the generators of this enlarged Poincaré group are given by

$$
P_{A}=-i \frac{\partial}{\partial y^{A}}, \quad S_{A B}=-i\left(\eta_{A C} y^{C} \frac{\partial}{\partial y^{B}}-\eta_{B C} y^{C} \frac{\partial}{\partial y^{A}}\right)
$$

Therefore, besides the two Poincaré symmetries there are other symmetries intertwining the space of the $x^{\mu}$ with the one of the one spanned by $\xi^{\mu \nu}$ :

$$
S_{\mu \nu, \rho}=-i \sqrt{\frac{|z|}{2}}\left(\xi_{\mu \nu} \frac{\partial}{\partial x^{\rho}}-\sqrt{\frac{2}{|z|}} x_{\rho} \frac{\partial}{\partial \xi_{\mu \nu}}\right)
$$

which together with the Poincaré generators in $D$ and $D(D-1) / 2$ dimensions span the full algebra of the Poincare group in $D(D+1) / 2$ dimensions. This analysis corresponds to the following decomposition of the Poincaré algebra in $D(D+1) / 2$ dimensions:

$$
\text { Poincaré in } D \operatorname{dim} \oplus \text { Poincaré in } \frac{D(D-1)}{2} \operatorname{dim} \oplus S_{\mu \nu, \rho} \text {. }
$$

As said before, the generators $S_{\mu \nu, \rho}$ intertwine the $x^{\mu}$ with the $\xi^{\mu \nu}$-space. Or, said in other way, they intertwine two different representations of the original Lorentz group in $D$ dimensions, namely: $(1 / 2,1 / 2)$ and $(1,0) \oplus(0,1)$.

The analysis of the generators of the large Poincaré algebra in terms of the original Lorentz algebra in (2.8) is made explicit in the tables 1 and 2. Besides giving the details for $D=4$ we do the same for $D=3$. This case is of particular interest because all the dynamical variables are three-vectors: $x_{\mu}, \xi_{\mu}=\frac{1}{2} \epsilon_{\mu \nu \rho} \xi^{\mu \nu}, \eta_{\mu}$. 


\section{Dimensional reduction}

We will now introduce a further term in the action, Using the pull back of the MaurerCartan one-forms, of section 2,

$$
S=\int d \tau\left(L_{1}+L_{2}\right)=S_{1}+S_{2}
$$

where

$$
L_{1}=-M \sqrt{-\dot{x}_{\mu}^{2}-\frac{z}{2} \dot{\xi}_{\mu \nu}^{2}} \quad L_{2}=-\frac{1}{R^{2}} \sqrt{-\left(\dot{\eta}^{\mu}-\xi^{\mu \nu} \dot{x}_{\nu}\right)^{2}}
$$

The model contains an extra vector $\eta_{\mu}$ associated to the generator $Z_{\mu}$. Given the dimensions in mass of $x^{\mu}$ and $\xi^{\mu \nu}$, the dimension in mass of $\eta^{\mu}$ is given by:

$$
\left[\eta_{\mu}\right]=-2 \text {. }
$$

An interesting feature appears due the introduction of the term depending on the MC form $\Omega_{3}^{\mu}: \sqrt{-\left(\dot{\eta}^{\mu}-\xi^{\mu \nu} \dot{x}_{\nu}\right)^{2}}$. The Poincaré algebra in $D(D+1) / 2$, with many times, is explicitly broken to the Poincaré algebra in $D$ dimensions, but there is an emergent symmetry generated by $Z_{\mu}$ which was acting trivially on the lagrangian (3.1), Therefore this term gives rise automatically to a dimensional reduction from $D(D+1) / 2$ to $D$ dimensions. It should be noticed that the extra-dimensional variables $\xi^{\mu \nu}$, at difference with the usual models with extra dimensions, are not Lorentz scalars, but rather they belong to a nontrivial representation of the Lorentz group.

The action (4.1), besides the global invariance under the coadjoint Poincaré algebra, it is invariant under two diffeomorphisms, one associated to $S_{1}$ and the other to $S_{2}$. To study this point, let us consider the Euler-Lagrange derivatives of the total action

$$
\begin{aligned}
\frac{\delta S}{\delta x^{\mu}} & =-\frac{d}{d \tau}\left(M \frac{\dot{x}_{\mu}}{\sqrt{-\dot{x}_{\mu}^{2}-\frac{z}{2} \dot{\xi}_{\mu \nu}^{2}}}-F_{\rho} \xi_{. \mu}^{\rho}\right) \\
\frac{\delta S}{\delta \xi^{\mu \nu}} & =-\frac{d}{d \tau}\left(z M \frac{\dot{\xi}_{\mu \nu}}{\sqrt{-\dot{x}_{\mu}^{2}-\frac{z}{2} \dot{\xi}_{\mu \nu}^{2}}}\right)-\left(F_{\mu} \dot{x}_{\nu}-F_{\nu} \dot{x}_{\mu}\right) \\
\frac{\delta S}{\delta \eta^{\mu}} & =-\frac{d}{d \tau} F_{\mu}
\end{aligned}
$$

where

$$
F_{\mu}=\frac{1}{R^{2}} \frac{\left(\dot{\eta}_{\mu}-\xi_{\mu \rho} \dot{x}^{\rho}\right)}{\sqrt{-\left(\dot{\eta}^{\mu}-\xi^{\mu \nu} \dot{x}_{\nu}\right)^{2}}} .
$$

The Euler-Lagrange derivatives are invariant under coadjoint Poincaré transformations (2.6) and are not independent. In fact, we have two Noether identities (see for example [15-17]), the first one associated to the diff-invariance of the total action, and the second one to the diff-invariance of $S_{2}$ :

$$
\begin{aligned}
\dot{x}^{\mu} \frac{\delta S}{\delta x^{\mu}}+\frac{1}{2} \dot{\xi}^{\mu \nu} \frac{\delta S}{\delta \xi^{\mu \nu}}+\dot{\eta}^{\mu} \frac{\delta S}{\delta \eta^{\mu}} & =0 \\
\left(\dot{\eta}^{\mu}-\xi^{\mu \rho} \dot{x}_{\rho}\right) \frac{\delta S}{\delta \eta^{\mu}} & =0
\end{aligned}
$$


Therefore we have two gauge symmetries

$$
\begin{aligned}
\delta_{1} x_{\mu} & =\rho_{1} \dot{x}_{\mu} \\
\delta_{1} \xi_{\mu \nu} & =\rho_{1} \dot{\xi}_{\mu \nu} \\
\delta_{1} \eta_{\mu} & =\rho_{1} \dot{\eta}_{\mu} \\
\delta_{2} x_{\mu} & =\delta_{2} \xi_{\mu \nu}=0, \quad \delta_{2} \eta_{\mu}=\rho_{2}\left(\dot{\eta}_{\mu}-\xi_{\mu \rho} \dot{x}^{\rho}\right)
\end{aligned}
$$

where $\rho_{1}$ and $\rho_{2}$ are arbitrary functions of $\tau$. The transformation with parameter $\rho_{1}$ is the ordinary world-line diffeomorphism. We can check explicitly that these transformations leave the lagrangian (4.1) invariant up to a total derivative:

$$
\delta_{1} L=\frac{d}{d \tau}\left(\rho_{1} L\right)
$$

and

$$
\delta_{2} L_{1}=0, \quad \delta_{2} L_{2}=c \frac{d}{d \tau}\left(\rho_{2} L_{2}\right)
$$

It is convenient define the analogue of the proper time for this model as:

$$
d s=\sqrt{-\dot{x}_{\mu}^{2}-\frac{z}{2} \dot{\xi}_{\mu \nu}^{2}} d \tau
$$

Notice, that this is nothing but the proper time for the particle in $D(D+1) / 2$ dimensions. Of course, the choice of this parameter is equivalent to the gauge choice:

$$
\sqrt{-\dot{x}_{\mu}^{2}-\frac{z}{2} \dot{\xi}_{\mu \nu}^{2}}=1
$$

Using the expressions (4.4)-(4.6) and (4.8) inside (4.9) we get the identity:

$$
\frac{d x^{\mu}}{d s} L_{\mu}^{1}+\frac{1}{2} \frac{d \xi^{\mu \nu}}{d s} L_{\mu \nu}^{2} \equiv 0
$$

where

$$
\begin{aligned}
L_{\mu}^{1} & =M \frac{d}{d s} \frac{d x_{\mu}}{d s}-F_{\rho} \frac{d \xi_{. \mu}^{\rho}}{d s} \\
L_{\mu \nu}^{2} & =z M \frac{d}{d s} \frac{\xi_{\mu \nu}}{d s}+\left(F_{\mu} \frac{d x_{\nu}}{d s}-F_{\nu} \frac{d x_{\mu}}{d s}\right) .
\end{aligned}
$$

The equations of motion are obtained from the vanishing of the expressions (4.4), (4.5) and (4.6). Using $\dot{F}_{\mu}=0$ (from (4.6)) in eq. (4.4), the other two equations of motion can be written as

$$
L_{\mu}^{1}=0, \quad L_{\mu \nu}^{2}=0 .
$$

The identity (4.15) shows that the two equations of motion are not independent one from the other. 
There are two interesting limiting situations that we could consider:

1) $-R \rightarrow \infty$ with $z$ fixed and different from zero. In this case the action (4.1) reduces to the first term, that is the action of a free particle in $D(D+1) / 2$ dimensions.

$$
S=-\int d \tau M \sqrt{-\dot{x}_{\mu}^{2}}
$$

2) $-z \rightarrow 0$ with fixed $R$. This would correspond to the choice

$$
S=\int d \tau\left(-M \sqrt{\dot{x}_{\mu}^{2}}-\frac{1}{R^{2}} \sqrt{-\left(\dot{\eta}^{\mu}-\xi^{\mu \nu} \dot{x}_{\nu}\right)^{2}}\right)
$$

Then, the kinetic term for $\xi^{\mu \nu}$ vanishes and these variables become non-dynamical. From their variations we get

$$
\left(F_{\mu} \frac{d x_{\nu}}{d s}-F_{\nu} \frac{d x_{\mu}}{d s}\right)=0
$$

which has the solution

$$
F_{\mu}=f(\tau) \dot{x}_{\mu} .
$$

Then, from the condition that $F_{\mu}$ is a time-like vector, $F^{2}=-1 / R^{4}$, we get

$$
F_{\mu}=\frac{1}{R^{2}} \frac{\dot{x}_{\mu}}{\sqrt{-\dot{x}^{2}}}
$$

But $F_{\mu}$ is constant, therefore

$$
\frac{d}{d \tau} \frac{\dot{x}_{\mu}}{\sqrt{-\dot{x}^{2}}}=0
$$

which it is the equation of motion of a free particle.

A third possibilities is to send $R \rightarrow \infty$ and $z \rightarrow 0$ in a correlated way, namely $z \approx 1 / R^{3}$. This case will be discussed in detail in section 4.2

\subsection{Hamiltonian analysis and canonical action}

As we have seen, the model described in this section admits two gauge symmetries. Therefore we expect the presence of two first class constraints in the phase space. To this end, let us perform the hamiltonian analysis of (4.1).

We start by computing the canonical momenta

$$
\begin{aligned}
p_{\mu} & =M \frac{\dot{x}_{\mu}}{\sqrt{-\dot{x}_{\mu}^{2}-\frac{z}{2} \dot{\xi}_{\mu \nu}^{2}}}-\frac{1}{R^{2}} \frac{\left(\dot{\eta}_{\nu}-\xi_{\nu \rho} \dot{x}^{\rho}\right) \xi_{. \mu}^{\nu}}{\sqrt{-\left(\dot{\eta}^{\mu}-\xi^{\mu \nu} \dot{x}_{\nu}\right)^{2}}} \\
\pi_{\mu} & =\frac{1}{R^{2}} \frac{\left(\dot{\eta}_{\mu}-\xi_{\mu \rho} \dot{x}^{\rho}\right)}{\sqrt{-\left(\dot{\eta}^{\mu}-\xi^{\mu \nu} \dot{x}_{\nu}\right)^{2}}} \equiv F_{\mu} \\
\pi_{\mu \nu} & =z M \frac{\dot{\xi}_{\mu \nu}}{\sqrt{-\dot{x}_{\mu}^{2}-\frac{z}{2} \dot{\xi}_{\mu \nu}^{2}}}
\end{aligned}
$$


where we have introduced the quantity $F_{\mu}$, as the momentum associated to $\eta^{\mu}$ in terms of the lagrangian variables.

From the previous expressions we see that there are two primary constraints

$$
\phi_{1}=\frac{1}{2}\left(\left(p_{\mu}+\pi_{\rho} \xi_{. \mu}^{\rho}\right)^{2}+\frac{1}{2 z} \pi_{\mu \nu}^{2}+M^{2}\right)=0, \quad \phi_{2}=\frac{1}{2}\left(\pi_{\mu}^{2}+\frac{1}{R^{4}}\right)=0 .
$$

These two constraints are first class:

$$
\left\{\phi_{1}, \phi_{2}\right\}=0
$$

Therefore there are no secondary constraints. Notice that in the two limits that we have previously considered, only the constraint $\phi_{1}$ survives. In the first case we get the massshell constraint for a particle in $D(D+1) / 2$ dimensions, whereas, in the second case we get the mass-shell condition for a particle in $D$ dimensions.

The presence of two first class constraints implies the existence of two gauge transformations given by

$$
\bar{\delta}_{i} A=\left\{A, \epsilon_{i} \phi_{i}\right\},
$$

where $\epsilon_{i}(\tau)$ are the gauge parameters. These transformations are the same as the ones given in (4.10) after the following identification of the parameters $\rho_{1}, \rho_{2}$

$$
\epsilon_{1}=\frac{1}{M} \rho_{1} \sqrt{-\dot{x}_{\mu}^{2}-\frac{z}{2} \dot{\xi}_{\mu \nu}^{2}}, \quad \epsilon_{2}=R^{2} \rho_{2} \sqrt{-\left(\dot{\eta}^{\mu}-\xi^{\mu \nu} \dot{x}_{\nu}\right)^{2}}
$$

with the exclusion of $\bar{\delta}_{1} \eta_{\mu}$ for which the variation is a combination of two of the gauge transformations of (4.10):

$$
\bar{\delta}_{1} \eta_{\mu}=\rho_{1} \xi_{\mu \rho} \dot{x}^{\rho}=\delta_{1} \eta_{\mu}-\frac{\rho_{1}}{\rho_{2}} \delta_{2} \eta_{\mu}
$$

Under these transformations we have

$$
\bar{\delta}_{1} L_{1}=M \frac{d}{d \tau}\left(\epsilon_{1} L_{1}\right), \quad \bar{\delta}_{1} L_{2}=0
$$

and

$$
\bar{\delta}_{2} L_{1}=0, \quad \bar{\delta}_{2} L_{2}=\frac{1}{R^{2}} \frac{d}{d \tau}\left(\epsilon_{2} L_{2}\right) .
$$

The canonical action is given by

$$
S_{c}=\int d \tau\left(p_{\mu} \dot{x}^{\mu}+\frac{1}{2} \pi_{\mu \nu} \dot{\xi}^{\mu \nu}+\pi_{\mu} \dot{\eta}^{\mu}-e_{1} \phi_{1}-e_{2} \phi_{2}\right)
$$

The degrees of freedom in phase space are $2 D$ for $x^{\mu}, p_{\mu}, D(D-1)$ for $\xi^{\mu \nu}, \pi_{\mu \nu}$ and $2 D$ for $\eta^{\mu}, \pi_{\mu}$. The physical degrees of freedom will be $2 D+D(D-1)+2 D-2 \times 2=D^{2}+3 D-4$, where we used the fact that there are two first class constraints. 


\subsection{The effective action}

In this section we would like to consider the limit $R \rightarrow \infty$ and $z \rightarrow 0$ as $z \rightarrow 1 / R^{\gamma}, \gamma>0$. We will integrate out the variables $\xi^{\mu \nu}$ at the first non vanishing order in $1 / R$. obtaining an effective action which, at the lowest order describes a free particle in $D$ dimensions, whereas at the next order the equations of motion describe a particle in a quadratic potential, that could be also interpreted as a correction to the Minkowski flat metric. The quadratic potential involves only the coordinates orthogonal to the time-like constant vector $F^{\mu}$ defined in (4.7). This effective action breaks the coadjoint Poincaré symmetry in $D$ space time dimensions. The effective action describes a particle in $D$ dimensions subject to a harmonic motion, for $z<0$.

In order to obtain an effective action from the decoupling of the extra dimensions, we will consider the equations of motion for $x^{\mu}$ and $\xi^{\mu \nu}$ in an arbitrary gauge and using $\dot{F}^{\mu}=0$

$$
\begin{aligned}
\frac{d}{d \tau}\left(M \frac{\dot{x}_{\mu}}{\sqrt{-\dot{x}_{\mu}^{2}-\frac{z}{2} \dot{\xi}_{\mu \nu}^{2}}}\right) & =F_{\rho} \dot{\xi}^{\rho}{ }_{\mu} \\
\frac{d}{d \tau}\left(z M \frac{\dot{\xi}_{\mu \nu}}{\sqrt{-\dot{x}_{\mu}^{2}-\frac{z}{2} \dot{\xi}_{\mu \nu}^{2}}}\right) & =-\left(F_{\mu} \dot{x}_{\nu}-F_{\nu} \dot{x}_{\mu}\right) .
\end{aligned}
$$

Let us introduce the coordinates longitudinal and transverse to $F^{\mu}$,

$$
x^{L}=\frac{F \cdot x}{\sqrt{-F^{2}}}, \quad x_{\mu}^{T}=x_{\mu}-F_{\mu} \frac{F \cdot x}{F^{2}},
$$

implying $x^{2}=-\left(x^{L}\right)^{2}+\left(x^{T}\right)^{2}$. Making use of $\dot{F}^{\mu}=0$, we integrate eq. (4.37) obtaining

$$
z M \frac{\dot{\xi}_{\mu \nu}}{\sqrt{-\dot{x}_{\mu}^{2}-\frac{z}{2} \dot{\xi}_{\mu \nu}^{2}}}=-\left(F_{\mu} x_{\nu}^{T}-F_{\nu} x_{\mu}^{T}+\alpha_{\mu \nu}\right)
$$

where

$$
\alpha_{\mu \nu}=-\left.z M \frac{\dot{\xi}_{\mu \nu}}{\sqrt{-\dot{x}_{\mu}^{2}-\frac{z}{2} \dot{\xi}_{\mu \nu}^{2}}}\right|_{\tau=0}-\left(F_{\mu} x_{\nu}^{T}(0)-F_{\nu} x_{\mu}^{T}(0)\right) .
$$

In order to integrate out completely the dependence on the $\xi$ variables, we need to eliminate in $\alpha_{\mu \nu}$ the dependence on the initial condition on $\dot{\xi}_{\mu \nu}$. This can only be done assuming that the first term in the expression of $\alpha_{\mu \nu}$ vanishes in the limit we are considering. Therefore, we will require that for $R \rightarrow \infty$, the parameter $z$ goes to zero faster than the second term, that is: $z \rightarrow 1 / R^{3}$. Then the eq. (4.39) can be written as:

$$
z M \frac{\dot{\xi}_{\mu \nu}}{\sqrt{-\dot{y}^{2}-\frac{z}{2} \dot{\xi}^{2}}}=-\left(F_{\mu} y_{\nu}^{T}-F_{\nu} y_{\mu}^{T}\right)
$$

with

$$
y_{\mu}=x_{\mu}-x_{\mu}(0) .
$$


Squaring the eq. (4.41) we get

$$
\dot{\xi}^{2}=-\frac{T^{2}}{z^{2} M^{2}} \frac{\dot{y}^{2}}{1+T^{2} /\left(2 z M^{2}\right)}
$$

where

$$
T_{\mu \nu}=-\left(F_{\mu} y_{\nu}^{T}-F_{\nu} y_{\mu}^{T}\right)
$$

from which

$$
\dot{x}^{2}+\frac{z}{2} \dot{\xi}^{2}=\dot{y}^{2}+\frac{z}{2} \dot{\xi}^{2}=\frac{1}{1+T^{2} /\left(2 z M^{2}\right)} \dot{y}^{2} .
$$

It is convenient to define

$$
W\left(y^{T}\right)=\frac{1}{1+T^{2} /\left(2 z M^{2}\right)}=\frac{1}{1-\left(y^{T}\right)^{2} /\left(z M^{2} R^{4}\right)} .
$$

Substituting into the equation of motion for $x_{\mu},(4.36)$, we get

$$
\frac{1}{\sqrt{-W \dot{y}^{2}}} \frac{d}{d \tau} \frac{\dot{y}_{\mu}}{\sqrt{-W \dot{y}^{2}}}-\frac{1}{z M^{2} R^{4}} y_{\mu}^{T}=0 .
$$

If we introduce the gauge fixing $\sqrt{-W \dot{y}^{2}}=1$, the equations of motion on this gauge become

$$
\ddot{y}^{\mu}-\frac{1}{z M^{2} R^{4}} y_{\mu}^{T}=0
$$

which for $z<0$ represents a harmonic motion in the transverse coordinates.

The diff invariant equation of motion (4.47) can be obtained from the action

$$
S=-M \int d \tau \sqrt{-\left(1-\frac{\left(y^{T}\right)^{2}}{z M^{2} R^{4}}\right) \dot{y}^{2}} .
$$

Notice that the action (4.49) can be interpreted as the action of a particle in a background metric given by

$$
g_{\mu \nu}=\left(1-\frac{\left(y^{T}\right)^{2}}{z M^{2} R^{4}}\right) \eta_{\mu \nu}
$$

The Christoffel symbols from this metric are given by

$$
\Gamma_{\mu \nu}^{\sigma}=-\frac{1}{z M^{2} R^{4}}\left(y_{\mu}^{T} \delta_{\nu}^{\sigma}+y_{\nu}^{T} \delta_{\mu}^{\sigma}-\left(y^{T}\right)^{\sigma} \eta_{\mu \nu}\right) .
$$

In order to evaluate the Riemann tensor we notice that the quadratic term in the Christoffel symbols is of higher order in $1 / R$ and therefore can be neglected:

$$
R_{\rho \mu \nu}^{\sigma} \approx \partial_{\nu} \Gamma_{\mu \rho}^{\sigma}-\partial_{\mu} \Gamma_{\nu \rho}^{\sigma}=-\frac{1}{z M^{2} R^{4}}\left(P_{\nu \rho} \delta_{\mu}^{\sigma}-P_{\mu \rho} \delta_{\nu}^{\sigma}+P_{\mu}^{\sigma} \eta_{\nu \rho}-P_{\nu}^{\sigma} \eta_{\mu \rho}\right)
$$

where

$$
P_{\mu \nu}=\eta_{\mu \nu}-\frac{F_{\mu} F_{\nu}}{F^{2}}
$$

Then, we have

$$
R_{\mu \nu}=R_{\mu \rho \nu}^{\rho}=-\frac{1}{z M^{2} R^{4}}\left((D-1) \eta_{\mu \nu}+(D-2) P_{\mu \nu}\right)
$$


and, for the scalar curvature:

$$
R_{\mu}^{\mu}=-\frac{2}{z M^{2} R^{4}}(D-1)^{2}
$$

In the particular reference frame $F^{\mu}=\left(1 / R^{2}, \overrightarrow{0}\right)$, we get

$$
R_{00}=+\frac{1}{z M^{2} R^{4}}(D-1), \quad R_{i j}=-\frac{(2 D-3)}{z M^{2} R^{4}} \delta_{i j} .
$$

Notice also that the square mass term associated to the extra variables in the original formulation is $z M^{2}$. Therefore this gravitational field is entirely dependent on the extra dimensional space.

As we have shown at the lowest order in $1 / R$ we get a particle moving in a quadratic potential in the transverse variables $y^{T}$. However, the action depends on a constant vector $F_{\mu}$ that,at this level, should be thought as a given vector. Correspondingly the Lorentz invariance and the spatial translations are broken. On the other hand the action is invariant under a diffeomorphism, therefore we expect a first class constraint. In fact, from

$$
p_{\mu}=M \sqrt{1-\frac{\left(y^{T}\right)^{2}}{z M^{2} R^{4}}} \frac{\dot{y}_{\mu}}{\sqrt{-\dot{y}^{2}}}
$$

we get

$$
p^{2}+M^{2}\left(1-\frac{\left(y^{T}\right)^{2}}{z M^{2} R^{4}}\right)=0 .
$$

At the lowest order we can evaluate the shift in mass due to the perturbation originating from the extra coordinates; let us put

$$
p^{L}=M+\epsilon .
$$

Inserting this expression in the mass shell condition we get

$$
\epsilon=\frac{1}{2 M}\left(p^{T}\right)^{2}-\frac{1}{2 z M R^{4}}\left(y^{T}\right)^{2} .
$$

Therefore, if $z<0$, from the quantum point of view this means that there are no negative energy states, and the shift in mass is given by

$$
\epsilon=\hbar \omega(n+1 / 2)
$$

with

$$
\omega^{2}=\frac{1}{|z| M^{2} R^{4}} .
$$

Notice that we could have started from the Klein-Gordon equation associated to the mass-shell constraint (4.58) and deriving the energy eigenvalues. The result would have been $E_{n}^{2}=M^{2}+2 M \hbar \omega(n+1 / 2)$. Expanding the positive energy solution at the order $1 / R$, we find that the correction to $p^{L}$ is the one in (4.61).

Summing up we have seen that starting from the original action (4.1), invariant under coadjoint Poincaré transformations, and integrating out the variables $\xi^{\mu \nu}$ (the variables associated to the extra dimensions), the associated effective action (4.49) describes for $z<0$ a harmonic oscillator. 


\section{Conclusions and outlook}

In this paper we have considered a dynamical model based on a non-linear representation of the coadjoint Poincaré group, which has a natural interpretation in terms of extra dimensions. At difference with the models of this sort, here the extra dimensions are described by an antisymmetric tensor with respect to the Lorentz group and contains many times.

Given our space-time in $D$ dimensions, the total space has $D(D+1) / 2$ dimension, meaning 10 dimensions for $D=4$. The dimensional reduction from $D(D+1) / 2$ dimensions to $D$ is performed through a term coupling together the coordinates $x^{\mu}$ and $\xi^{\mu \nu}$, This term introduces another vector $\eta^{\mu}$ giving rise to a constant time-like vector, $F^{\mu}$ (the momentum conjugated to $\eta^{\mu}$ ), but always maintaining the invariance under the coadjoint Poincaré symmetry.

We have studied the model in a particular limit, where it is possible to integrate out the extra dimensional coordinates. The effective action obtained in this way describes a particle in $D$ dimension moving in a quadratic potential in the $D-1$ space orthogonal to the time-like vector $F^{\mu}$. In the case of z negative, in a first quantised version, this gives rise to a mass spectrum of a harmonic oscillator. The quadratic potential has an interesting interpretation in terms of a modification to the Minkowski flat metrics. The resulting metrics depends on the constant time-like vector $F^{\mu}$ and has non-vanishing Riemann, Ricci tensor and scalar curvature.

In practice we have shown that the existence of extra dimensions, with non trivial behaviour with respect to the Lorentz group, is capable to modify the geometry of our space-time.

We think that this example might offer new interesting possibilities for constructing new models of theories with extra dimensions and about the relative dimensional reduction.

About this point, it will be interesting to study the extension of this model to $p$-branes. Using the pull-back of the MC forms in eq. (2.5) we can construct an action invariant under the coadjoint Poincaré algebra for strings and more generally for $p$-branes. For instance we can consider the string extension of the diff-invariant model for a point particle considered previously. We will describe the strings in the Polyakov formulation with a metric in the world-sheet [18]. ${ }^{1}$ All the dynamical variables are functions of the two variables $(\sigma, \tau)$ which are also denoted by $\xi^{\alpha}, \alpha=1,2$. We will introduce also the following quantities

$$
\gamma_{\alpha \beta}^{1}=\frac{\partial x^{\mu}}{\partial \xi^{\alpha}} \frac{\partial x_{\mu}}{\partial \xi^{\beta}}, \quad \gamma_{\alpha \beta}^{2}=\frac{1}{2} \frac{\partial \xi^{\mu \nu}}{\partial \xi^{\alpha}} \frac{\partial \xi_{\mu \nu}}{\partial \xi^{\beta}}, \quad \gamma_{\alpha \beta}^{3}=\left(\frac{\partial \eta^{\mu}}{\partial \xi^{\alpha}}-\xi^{\mu \rho} \frac{\partial x_{\rho}}{\partial \xi^{\alpha}}\right)\left(\frac{\partial \eta_{\mu}}{\partial \xi^{\alpha}}-\xi_{\mu \sigma} \frac{\partial x^{\sigma}}{\partial \xi^{\alpha}}\right)
$$

Then, the Polyakov action analogous to the one that we have used for the point particle is

$$
S=-\frac{1}{4 \pi \alpha^{\prime}} \int d \sigma d \tau \sqrt{-h} h^{\alpha \beta}\left(\gamma_{\alpha \beta}^{1}+z \gamma_{\alpha \beta}^{2}\right)-\frac{1}{4 \pi \beta^{\prime}} \int d \sigma d \tau \sqrt{-h} h^{\alpha \beta} \gamma_{\alpha \beta}^{3}
$$

\footnotetext{
${ }^{1}$ We will use the notations of Zwiebach's book [18].
} 
where $h^{\alpha \beta}$ is an arbitrary two-dimensional metric, $h=\operatorname{det}\left(h_{\alpha \beta}\right)$. Eliminating the zweibeins via their equations of motion, one gets:

$$
S=-\frac{1}{2 \pi \alpha^{\prime}} \int d \sigma d \tau \sqrt{-\operatorname{det}\left(\gamma_{\alpha \beta}^{1}+z \gamma_{\alpha \beta}^{2}\right)}-\frac{1}{2 \pi \beta^{\prime}} \int d \sigma d \tau \sqrt{-\operatorname{det}\left(\gamma_{\alpha \beta}^{3}\right)} .
$$

This action can be easily generalised to the action of a $p$-brane. For this, it is sufficient to go from a 2-dimensional world-sheet to a $p+1$ dimensional, implying $\alpha, \beta=1, \cdots, p+1$ and to replace the volume element

$$
d \sigma d \tau \rightarrow d \xi_{1} \cdots d \xi_{p+1}
$$

Also in this case, neglecting the second term in the action, we obtain the action for a $D(D+1) / 2$ dimensional string (or $p$-brane). In the case of $D=4$ a 10 -dimensional extended object.

\section{Acknowledgments}

We acknowledge discussions with Axel Kleinschmidt and Patricio Salgado-Rebolledo. JG has been supported in part by MINECO FPA2016-76005-C2-1-P and Consolider CPAN, and by the Spanish government (MINECO/FEDER) under project MDM-2014-0369 of ICCUB (Unidad de Excelencia María de Maeztu).

Open Access. This article is distributed under the terms of the Creative Commons Attribution License (CC-BY 4.0), which permits any use, distribution and reproduction in any medium, provided the original author(s) and source are credited.

\section{References}

[1] J. Gomis and H. Ooguri, Nonrelativistic closed string theory, J. Math. Phys. 42 (2001) 3127 [hep-th/0009181] [INSPIRE].

[2] U.H. Danielsson, A. Guijosa and M. Kruczenski, IIA/B, wound and wrapped, JHEP 10 (2000) 020 [hep-th/0009182] [inSPIRE].

[3] J. Brugues, T. Curtright, J. Gomis and L. Mezincescu, Non-relativistic strings and branes as non-linear realizations of Galilei groups, Phys. Lett. B 594 (2004) 227 [hep-th/0404175] [INSPIRE].

[4] J. Gomis, J. Gomis and K. Kamimura, Non-relativistic superstrings: A New soluble sector of $A d S_{5} \times S^{5}, J H E P 12(2005) 024$ [hep-th/0507036] [INSPIRE].

[5] J. Brugues, J. Gomis and K. Kamimura, Newton-Hooke algebras, non-relativistic branes and generalized pp-wave metrics, Phys. Rev. D 73 (2006) 085011 [hep-th/0603023] [INSPIRE].

[6] A. Barducci, R. Casalbuoni and J. Gomis, Confined dynamical systems with Carroll and Galilei symmetries, Phys. Rev. D 98 (2018) 085018 [arXiv:1804.10495] [INSPIRE].

[7] A. Barducci, R. Casalbuoni and J. Gomis, Non-relativistic k-contractions of the Coadjoint Poincaré algebra, Int. J. Mod. Phys. A 35 (2020) 2050009 [arXiv:1910.11682] [INSPIRE].

[8] E. Bergshoeff, J. Gomis and P. Salgado-ReboLledó, Non-relativistic limits and three-dimensional coadjoint Poincaré gravity, arXiv:2001.11790 [INSPIRE]. 
[9] G. Papageorgiou and B.J. Schroers, A Chern-Simons approach to Galilean quantum gravity in $2+1$ dimensions, JHEP 11 (2009) 009 [arXiv:0907.2880] [INSPIRE].

[10] E.A. Bergshoeff and J. Rosseel, Three-Dimensional Extended Bargmann Supergravity, Phys. Rev. Lett. 116 (2016) 251601 [arXiv: 1604.08042] [INSPIRE].

[11] J. Hartong, Y. Lei and N.A. Obers, Nonrelativistic Chern-Simons theories and three-dimensional Hořava-Lifshitz gravity, Phys. Rev. D 94 (2016) 065027 [arXiv: 1604.08054] [INSPIRE].

[12] N. Ozdemir, M. Ozkan, O. Tunca and U. Zorba, Three-Dimensional Extended Newtonian (Super)Gravity, JHEP 05 (2019) 130 [arXiv: 1903.09377] [INSPIRE].

[13] V. Bargmann, On Unitary ray representations of continuous groups, Annals Math. 59 (1954) 1 [INSPIRE].

[14] S. Bonanos and J. Gomis, Infinite Sequence of Poincaré Group Extensions: Structure and Dynamics, J. Phys. A 43 (2010) 015201 [arXiv:0812.4140] [inSPIRE].

[15] I.A. Batalin and G.A. Vilkovisky, Existence Theorem for Gauge Algebra, J. Math. Phys. 26 (1985) 172 [INSPIRE].

[16] M. Henneaux and C. Teitelboim, Quantization of gauge systems, Cambridge University Press (1992).

[17] J. Gomis, J. Paris and S. Samuel, Antibracket, antifields and gauge theory quantization, Phys. Rept. 259 (1995) 1 [hep-th/9412228] [INSPIRE].

[18] B. Zwiebach, A first course in string theory, Cambridge University Press (2006). 